\title{
Reporte de cinco casos de malaria neonatal grave por Plasmodium vivax en Urabá, Colombia
}

\author{
Juan Gabriel Piñeros-Jiménez ${ }^{1}$, Margarita Arboleda ${ }^{2}$, Juan Camilo Jaramillo ${ }^{1}$, Silvia Blair ${ }^{1}$ \\ ${ }^{1}$ Grupo de Malaria, Universidad Antioquia, Medellín, Colombia \\ 2 Instituto Colombiano de Medicina Tropical "Antonio Roldán Betancur”, ICMT-CES, Medellín, Colombia
}

Introducción. La malaria neonatal es aquélla que ocurre durante el primer mes de vida. Durante más de medio siglo se reporta la presencia de parásitos de malaria en neonatos, generalmente asociada a transmisión congénita, cuya frecuencia se ha incrementado desde hace algunos años, especialmente en África. En Latinoamérica su situación es desconocida y sólo hay reportes aislados de casos.

Objetivos. Describir los casos de paludismo neonatal diagnosticado en los hospitales de la región del Urabá antioqueño entre marzo de 2002 y marzo de 2004.

Materiales y métodos. Se hizo una búsqueda de casos en los hospitales de Turbo y Apartadó que cumplieran los criterios de malaria neonatal. Se buscaron y tabularon datos sobre algunas características: maternas, demográficas de los neonatos, clínicas de la enfermedad y hallazgos de laboratorio.

Resultados. Se encontraron cinco casos de malaria neonatal, todos por Plasmodium vivax, de los cuales, sólo uno cumplió los criterios de infección congénita. Aunque tres de los pacientes tuvieron parto institucional y dos antecedentes maternos de malaria gestacional, ninguno fue tamizado para malaria; $25 \%$ (1 de 4) de las madres eran primíparas y la mitad eran menores de 20 años. Todos los neonatos tuvieron fiebre, algún signo de enfermedad grave al examen físico de ingreso y cifras de hemoglobina compatibles con anemia neonatal grave. Ninguno recibió el esquema antipalúdico recomendado.

Conclusión. Se trata de un reporte de cinco casos de malaria neonatal grave por $P$. vivax, especie que habitualmente no se relaciona con complicaciones, sin que existiera en ningún caso la sospecha clínica y con tratamiento inadecuado.

Palabras clave: Plasmodium vivax, malaria paludismo, recién nacido, estudios de casos, Colombia.

\section{Report of five cases of severe neonatal Plasmodium vivax malaria in Urabá, Colombia}

Introduction. Neonatal malaria is a type of malaria that occurs during the first month of life. In the last half century, the reports of malaria parasites in neonates generally have been associated with congenital transmission. However, in recent years, cases have appeared with increasing frequency, especially in Africa. In Latin America, the incidence of neonatal malaria is unknown, with only isolated cases reported.

Objective. Cases of neonatal malaria were identified and characterized to better recognize the frequency and symptoms of cases as they occur in Colombia.

Materials and methods. Between March 2002-March 2004, a search for cases of neonatal malaria was made in the hospitals of the Turbo and Apartadó counties (Urabá, Antioquia Province). The following date were compiled: (1) characteristics of the mother, (2) demographic characteristics of the neonates, (3) clinical characteristics of the disease, and (4) laboratory results.

Results. Five cases were discovered of neonatal vivax malaria; however, only one met the criteria for congenital infection. Three patients had institutional delivery and two had a maternal history of gestational malaria, but none underwent a screening test for malaria. One of the four mothers were primaparous and half of them were younger than 20 years. All neonates had fever and presented some sign of severe disease during the first medical examination; each 
had hemoglobin levels compatible with severe neonatal anemia. No neonate had received the recommended treatment for this type of malaria.

Conclusion: Five cases of severe neonatal malaria were reported, caused by infections of $P$. vivax, which normally does not produce severe disease. Since none of the malaria cases were recognized or treated at the local hospitals, advisories to medical professionals are recommended concerning neonatal malaria, particularly in endemic regions.

Key words: Plasmodium vivax, malaria; infant, newborn, case studies, Colombia.

La malaria, o paludismo, es la enfermedad parasitaria de mayor impacto sobre la salud de las poblaciones y afecta principalmente a los menores de cinco años (1). Sin embargo, su frecuencia durante el período neonatal es baja y sus características clínicas en esta etapa son poco conocidas. En Antioquia, la Dirección Seccional de Salud reportó que 0,6\% (196/31.559) de los casos de malaria a nivel departamental se presentaron en niños menores de un año, durante el año 2005 (2).

Desde hace más de cincuenta años se reporta la presencia de Plasmodium sp. en neonatos, la cual se atribuye principalmente a la transmisión vertical (3) . En los últimos años, ésta se incrementó sobre todo en países africanos de alta endemia donde encontraron frecuencias altas de parasitemia neonatal. En 1997, Obiajunwa y colaboradores, al estudiar 120 neonatos nacidos de 115 mujeres que habían sufrido malaria durante la gestación, encontraron $46,7 \%$ de muestras de sangre neonatal positiva para Plasmodium sp. (4). Asimismo, en el 2004, Orogade encontró una prevalencia de $8,3 \%(17 / 206)$ de malaria neonatal en el Hospital Universitario de Abu en Nigeria y Runsewe-Abiodun, en el 2006, reportó una prevalencia de $24,8 \%(5,6)$.

El paludismo neonatal es el que se presenta durante el primer mes de vida, cuyos diversos mecanismos de transmisión deben procurar distinguir entre aquéllos de tipo vectorial, hospitalario y congénito, dado el diferente tipo de

\footnotetext{
Correspondencia:

Juan Gabriel Piñeros-Jiménez, Grupo de Malaria, Universidad de Antioquia, Sede de Investigación Universitaria, Calle 62 No 52-59, laboratorio 610-611, Medellín, Colombia. Apartado aéreo 1226.

Telefax: (574) 210-6487.

malaria@quimbaya.udea.edu.co

Recibido: 03/03/08; aceptado:12/08/08
}

acciones dirigidas a su control. Entre éstos, el congénito cobra una gran importancia debido a su íntima relación con las malarias durante la gestación y placentaria.

En Latinoamérica, en general, el panorama de la malaria neonatal es desconocido. Actualmente sólo existen algunos reportes de casos de malaria congénita realizados en el Brasil y un estudio descriptivo prospectivo realizado por Fernández y colaboradores en Honduras, en el que reportaron una frecuencia de $3,3 \%$ de paludismo en neonatos nacidos de madres con antecedente de esta enfermedad durante el embarazo (7-9). En Colombia, a la fecha se han reportado 12 casos, todos relacionados con transmisión congénita y ninguno desde 1986 (10).

En el presente reporte se describen cinco casos de malaria neonatal por $P$. vivax atendidos en los hospitales de los municipios de Apartadó y Turbo (Urabá antioqueño) entre marzo de 2002 y marzo de 2004, en los cuales se hace énfasis en los hallazgos clínicos encontrados en estos pacientes por la presencia de enfermedad grave.

\section{Materiales y métodos}

La búsqueda de los neonatos se hizo de manera retrospectiva en los archivos de los hospitales locales de los municipios de Turbo y Apartadó, en el período antes señalado, y el único criterio de inclusión fue que el inicio de la enfermedad actual referido por la madre, ocurriera durante los primeros 28 días de vida postnatal, de acuerdo con el criterio de la Organización Mundial de la Salud (11), aunque el diagnóstico de paludismo fuera realizado algunos días después de este período.

La búsqueda se inició en los archivos de los hospitales locales, en cuyas bases de datos se indagó por los casos de malaria ocurridos durante los primeros dos meses de vida, a quienes se les 
aplicó el criterio de inclusión antes señalado. Se encontraron 13 pacientes con paludismo en los primeros dos meses de vida, de los cuales, sólo cinco cumplieron el criterio de inclusión de este reporte. De ellos, se utilizó la información proveniente de sus historias clínicas y sus respectivos resultados de laboratorios. Se tabularon dichos datos en una hoja de cálculo y se hicieron descripciones de variables demográficas, clínicas, de laboratorio, tratamientos y antecedentes maternos. Se encontraron las limitaciones propias de utilizar fuentes secundarias, en las cuales la mayoría de veces los datos son incompletos y poco uniformes entre los pacientes.

\section{Resultados}

\section{Características de los neonatos y sus madres}

Se encontraron cinco casos de paludismo diagnosticado en neonatos a término, en los cuales sólo se reportó infección por $P$. vivax. Tres de los cincop casos correspondió a pacientes de sexo femenino. Dos de los pacientes residían en el municipio de Turbo, dos en Apartadó y uno en Carepa, de los cuales, tres provenían de zona rural. Dos de los casos fueron producto de un embarazo gemelar. La edad promedio de los pacientes en el momento del diagnóstico de malaria fue de 30,4 días. En el cuadro 1 se resumen estos aspectos.

Entre los antecedentes maternos más relevantes encontramos una edad promedio 20,4 años; llama la atención que dos de las cuatro madres eran menores de 18 años. Una de las maternas era primigestante. Dos de ellas tuvieron antecedente de malaria durante la gestación por $P$. vivax una en una ocasión y, la otra, en tres, ambas durante el tercer trimestre de gestación; la materna que tuvo más de un antecedente refirió que uno de estos fue en el segundo trimestre.

Todas recibieron tratamiento antipalúdico con 25 $\mathrm{mg} / \mathrm{kg}$ de cloroquina durante tres días y la madre que presentó tres cuadros de malaria en el embarazo tuvo que ser transfundida en el segundo episodio por presentar anemia grave. Las dos madres restantes tuvieron antecedente de fiebre intermitente durante el embarazo, desde el sexto mes de gestación en una, y desde el octavo mes, en la otra; ninguna consultó por este motivo y el diagnóstico de paludismo se hizo posterior al parto. Sólo dos de las madres asistieron a controles prenatales. La mitad de los partos fue domiciliario y el mismo porcentaje requirió cesárea, uno por antecedente de cesárea previa y otro por embarazo gemelar (cuadro 2).

\section{Descripción del cuadro clínico y hallazgos de laboratorio}

La enfermedad actual de los neonatos se caracterizó por tener una duración promedio de 11 días, e iniciarse a una edad media de 20,4 días. La fiebre fue el motivo de consulta en todos los casos. Otros síntomas referidos fueron palidez $(60 \%)$, deposiciones diarreicas, tos y rinorrea $(40 \%)$, e irritabilidad, vómito y epistaxis (20\%).

En el examen físico de ingreso presentaron intensa palidez y hepatoesplenomegalia en todos los casos, acompañadas de soplo sistólico e ictericia en un caso cada uno. En tres pacientes se encontró fiebre al examen de ingreso (temperatura axilar $>37,2^{\circ} \mathrm{C}$ ) y en otro se halló hipotermia (temperatura axilar $<36^{\circ} \mathrm{C}$ ). Un paciente presentó dificultad respiratoria con polipnea (frecuencia respiratoria de 70 por minuto) y

Cuadro 1. Características demográficas de los pacientes incluidos.

\begin{tabular}{llllll}
\hline Característica & Paciente 1* & Paciente 2* & Paciente 3 & Paciente 4 & Paciente 5 \\
\hline Edad (días) & 31 & 31 & 23 & 34 & 33 \\
Edad al inicio de síntomas (días) & 28 & 26 & 17 & 19 & 12 \\
Sexo & Masculino & Femenino & Femenino & Femenino & Masculino \\
Municipio de residencia & Turbo & Turbo & Carepa & Apartadó & Apartadó \\
Zona de residencia & Rural & Rural & Rural & Urbana & Urbana \\
\hline
\end{tabular}

* Los pacientes 1 y 2 eran gemelos. 
cianosis, aunque no requirió asistencia respiratoria. Un resumen de la enfermedad actual se presenta en el cuadro 3.

Se reportaron en todos los pacientes valores de hemoglobina inferiores a $7 \mathrm{~g} / \mathrm{dl}$ (promedio de 5,08 $\mathrm{g} / \mathrm{dl}$ ) y valores de hematocrito inferiores a $20 \%$ (promedio de 14,66\%). Igualmente, en todos los casos el recuento plaquetario fue inferior a $150.000 / \mu l$ (promedio de $87.200 / \mu \mathrm{l}$ ) y en el $60 \%$ dicho recuento fue inferior a $100.000 / \mu l$. Se midieron los valores de bilirrubina total y directa en 3 de los 5 pacientes y se encontraron cifras promedio de 2,89 y $1,0 \mathrm{mg} / \mathrm{dl}$, respectivamente. En el cuadro 4 se resumen los hallazgos de laboratorio.

En 3 de los 5 de los casos hubo diagnóstico de paludismo al ingreso a los servicios de urgencias; en dos casos el diagnóstico se realizó por gota gruesa y por el microscopista, y en otro caso el diagnóstico fue hecho por extendido de sangre periférica de manera casual. Un caso tuvo diagnóstico inicial de neumonía y fue tratado inicialmente con una combinación antibiótica empírica de amplio espectro. El medicamento antipalúdico de base utilizado en todos los casos fue cloroquina. Dos de los pacientes recibieron al ingreso tratamiento con la combinación quinina más sulfadoxina/pirimetamina y luego se continuó con cloroquina. Al contrario, a otro se le adicionó quinina por persistir con parasitemia el día 3, al considerarse que se trataba de una falla terapéutica. El $80 \%$ de los pacientes (4/5) recibió transfusión de glóbulos rojos empaquetados. Uno de los pacientes presentó taquipnea y taquicardia el día 3 de hospitalización y requirió oxigenoterapia. La estancia hospitalaria promedio de estos pacientes fue de 5,4 días.
A continuación se presenta una breve descripción de cada uno de los casos de este reporte.

\section{Paciente 1}

Se trata de un paciente de sexo masculino de 31 días de edad, procedente de la vereda Claudia María del corregimiento El Dos del municipio de Turbo; fue producto de embarazo gemelar de madre multigestante de 40 años, con antecedente de tres episodios de malaria por $P$. vivax durante el embarazo, dos de los cuales requirieron hospitalización, el último, dos meses antes del parto, el cual requirió transfusión de hemoderivados por anemia grave.

El neonato fue llevado al puesto de diagnóstico de malaria del corregimiento por presentar fiebre y palidez de tres días de evolución. Allí la microscopista realizó diagnóstico de paludismo por $P$. vivaxy, por ser neonato, lo remitió al hospital del municipio.

Al ingreso se encontró tranquilo, con palidez cutánea generalizada, palidez en palmas y plantas, temperatura axilar de $37,4^{\circ} \mathrm{C}$, frecuencia cardiaca de 158 por minuto y frecuencia respiratoria de 54 por minuto. En el examen de abdomen, se palparon el hígado y el bazo en la región umbilical.

Los resultados de los exámenes de laboratorio de ingreso fueron: hemoglobina, 5,2 g/dl, hematocrito, $15 \%$, recuento de leucocitos, $7.800 / \mu \mathrm{l}$, recuento de pla-quetas $76.000 / \mu \mathrm{l}$, bilirrubina total $3,15 \mathrm{mg} / \mathrm{dl}$ y bilirrubina directa 1,3 mg/dl. Debido a la palidez y los valores tan bajos de la hemoglobina, se consideró un paludismo grave y se inició tratamiento con quinina y sulfadoxina/pirimetamina; se remitió al hospital de referencia de la zona de Urabá para

Cuadro 2. Características de las madres de los pacientes.

\begin{tabular}{lcccc}
\hline Características & Materna 1* & Materna 2 & Materna 3 & Materna 4 \\
\hline Edad materna (años) & 40 & 16 & 17 & 36 \\
Número de partos de la madre & Multigestante & Multigestante & Primigestante & Secundigestante \\
Asistencia a control prenatal & Sí & Sí & No & No \\
Atención de parto & Institucional & Institucional & Domiciliario & Institucional \\
Tipo de parto & Cesárea & Vaginal & Vaginal & Cesárea \\
Antecedente de malaria durante la gestación & Sí & Sí & No & No \\
Número de episodios de malaria durante la gestación & 3 & 1 & No documentado & No documentado
\end{tabular}

* Madre de los pacientes 1 y 2 
Cuadro 3. Características clínicas de la enfermedad actual de los casos.

\begin{tabular}{lc} 
Tiempo de duración de la enfermedad actual: media: 11 días $(3-21)$ \\
Edad de inicio de la enfermedad actual: media: 20,4 días $(12-30)$ \\
\hline Frecuencia de manifestaciones clínicas en la enfermedad actual (\%) \\
Fiebre & 100 \\
Palidez & 60 \\
Diarrea & 40 \\
Tos & 40 \\
Rinorrea & 40 \\
Irritabilidad & 20 \\
Vómito & 20 \\
Epistaxis & 20 \\
Frecuencia de signos en el examen físico de ingreso (\%) \\
Palidez intensa & 100 \\
Hepatoesplenomegalia & 100 \\
Temperatura axilar $>37,2{ }^{\circ} \mathrm{C}$ & 60 \\
Ictericia & 20 \\
Soplo cardiaco & 20 \\
Temperatura axilar $<36,0^{\circ} \mathrm{C}$ & 20 \\
Dificultad respiratoria & 20
\end{tabular}

Cuadro 4. Hallazgos de laboratorio.

\begin{tabular}{|c|c|}
\hline Examen de laboratorio (valor normal) & Promedio (valor mínimo-máximo) \\
\hline Hemoglobina (g/dl) & $5,08(3,8-6,5)$ \\
\hline Hematocrito $(\%)$ & $14,66 \%(11,0-18,4)$ \\
\hline Recuento de leucocitos (leucocitos/ $\mu$ l) & $10.467(7.300-15.500)$ \\
\hline Recuento de plaquetas (plaquetas/ul) & $87.200(30.000-118.000)$ \\
\hline Bilirrubina total $^{*}(\mathrm{mg} / \mathrm{dl})$ & $2,89 \mathrm{mg} / \mathrm{dl}(2,2-3,32)$ \\
\hline Bilirrubina directa* (mg/dl) & $1,0 \mathrm{mg} / \mathrm{dl}(0,7-1,3)$ \\
\hline
\end{tabular}

* Datos de 3 pacientes

realizar transfusión de glóbulos rojos empaquetados. Allí se cambió tratamiento a monoterapia con cloroquina. Luego de cinco días de hospitalización y ante la mejoría clínica, se dio de alta.

\section{Paciente 2}

Se trata de una paciente de sexo femenino de 31 días de edad, hermana gemela del paciente número 1. Fue llevada al puesto de diagnóstico de malaria del corregimiento junto con su hermano, por cuadro de cinco días de fiebre y palidez. Allí se realizó diagnóstico con gota gruesa, la cual fue positiva para paludismo por $P$. vivaxy se remitió al hospital donde se encontró una paciente tranquila, con palidez cutánea generalizada, palidez en palmas y plantas, ictericia en escleras y mucosa oral, temperatura axilar de $35,5^{\circ} \mathrm{C}$, frecuencia cardiaca de 158 por minuto y frecuencia respiratoria de 56 por minuto.

En el examen del abdomen se palpó hepatoesplenomegalia. Los resultados de los exámenes paraclínicos de ingreso fueron: hemoglobina 3,9 $\mathrm{g} / \mathrm{dl}$, hematocrito $11,4 \%$, recuento de leucocitos $15.100 / \mu \mathrm{l}$, recuento de plaquetas $118.000 / \mu \mathrm{l}$, bilirrubina total $3,32 \mathrm{mg} / \mathrm{dl}$ y bilirrubina directa 1,0 $\mathrm{mg} / \mathrm{dl}$. Con estos resultados se diagnosticó anemia grave, se inició tratamiento antipalúdico con quinina y sulfadoxina/pirimetamina y se remitió al hospital regional para transfusión de glóbulos rojos empaquetados. En el hospital de referencia se cambió el tratamiento por monoterapia con cloroquina. Luego de cinco días de hospitalización y ante la mejoría clínica se dio de alta. 


\section{Paciente 3}

Se trata de una paciente de sexo femenino, de 23 días de edad, procedente de zona rural del municipio de Carepa, quien fue llevada al servicio de urgencias del hospital por fiebre de seis días de evolución y cuatro días de rinorrea hialina y deposiciones diarreicas sin moco ni sangre. El día anterior a la consulta presentó dos episodios de epistaxis.

Al ingreso se encontró hipoactiva, con palidez generalizada y signos epistaxis reciente en narina derecha, temperatura axilar de $37,5^{\circ} \mathrm{C}$, frecuencia cardiaca de 96 por minuto y frecuencia respiratoria de 32 por minuto. En el abdomen se encontró hepatoesplenomegalia. Con esta historia se hizo una impresión diagnóstica de dengue con manifestaciones hemorrágicas y se inició tratamiento con líquidos y antipiréticos.

Los exámenes paraclínicos de ingreso reportaron valores de hemoglobina $6,5 \mathrm{~g} / \mathrm{dl}$, hematocrito $18,4 \%$, recuento de leucocitos $8.600 / \mathrm{ml}$, recuento de plaquetas $30.000 / \mu \mathrm{l}$, bilirrubina total $2,2 \mathrm{mg} / \mathrm{dl}$ y bilirrubina directa $0,7 \mathrm{mg} / \mathrm{dl}$. A los dos días de hospitalización, la paciente persistió con fiebre por lo que se solicitó gota gruesa, la cual fue positiva para $P$. vivax. Con este resultado se inició tratamiento con cloroquina. La paciente presentó mejoría de su cuadro febril. A pesar de su estado hematológico, no se realizó ninguna intervención. Al sexto día se dio de alta.

\section{Paciente 4}

Se trata de una paciente de sexo femenino, de 34 días, procedente de zona urbana del municipio de Apartadó, quien había consultado cinco días antes por cuadro de fiebre intermitente, rinorrea y tos húmeda de 15 días de evolución. Se hizo diagnóstico de neumonía y se inició tratamiento ambulatorio con amoxicilina y Dimetapp $\AA$. A los cinco días, la paciente asistió a control y la madre refirió persistencia de los síntomas y aparición de palidez generalizada. Por este motivo fue remitida al hospital de referencia de la zona.

Al ingreso se encontró una paciente tranquila, hidratada, con temperatura axilar de $37^{\circ} \mathrm{C}$, frecuencia cardiaca de 96 por minuto y frecuencia respiratoria de 32 por minuto. En la exploración del abdomen se palpó hepatoesplenomegalia. En este momento se reinterrogó a la madre, quien refirió que desde el sexto mes de embarazo tuvo fiebre intermitente, subjetiva y leve, cada dos a tres días. Con este antecedente se tomaron exámenes de gota gruesa a ella y a su hija, que fueron reportadas como positivas para $P$. vivax.

Los exámenes de laboratorio al momento del diagnóstico mostraron valores de hemoglobina 6,0 $\mathrm{g} / \mathrm{dl}$, hematocrito $17,5 \%$, recuento de leucocitos $7.300 / \mu \mathrm{l}$ y recuento de plaquetas $113.000 / \mu \mathrm{l}$. Bajo estas circunstancias se decidió tratar con cloroquina y realizar transfusión de glóbulos rojos empaquetados. En el tercer día de hospitalización la paciente continuó presentando gota gruesa positiva, por lo que se consideró que tenía una falla terapéutica temprana y se inició tratamiento con sulfato de quinina. Al segundo día de administración de la quinina, la gota gruesa fue negativa. Cuatro días más tarde se dio de alta.

\section{Paciente 5}

Se trata de un paciente de sexo masculino, de 33 días de edad, procedente de zona periurbana del municipio de Apartadó, quien consultó al hospital de referencia para la zona de Urabá con un cuadro clínico de 21 días de evolución que consistía en fiebre intermitente e irritabilidad. Siete días antes de la consulta la fiebre se acompañó de palidez, tos y vómito.

Al ingreso se encontró un paciente decaído, con palidez intensa generalizada, polipneico, con cianosis peribucal, con temperatura de $37,8^{\circ} \mathrm{C}$, frecuencia cardiaca de 160 por minuto y frecuencia respiratoria de 70 por minuto. Al examen del tórax se auscultó un soplo cardiaco. En el abdomen se evidenció hepatoesplenomegalia. Por el estado clínico del paciente se inició un tratamiento urgente con oxigenoterapia por cámara cefálica y se solicitaron estudios paraclínicos.

Los resultados de los exámenes mostraron valores de hemoglobina 3,8 $\mathrm{g} / \mathrm{dl}$, hematocrito $11 \mathrm{~g} / \mathrm{dl}$, recuento de leucocitos $8.500 / \mu \mathrm{l}$ y recuento de plaquetas $99.000 / \mu \mathrm{l}$. El extendido de sangre periférica reportó formas compatibles con hemoparásitos de la especie $P$. vivax. Con estos resultados se decidió iniciar tratamiento específico 
con cloroquina y realizar transfusión de glóbulos rojos empaquetados, necesitándose dos de ellas. Se reinterrogó a la madre, quien afirmó que desde el octavo mes de embarazo presentaba fiebre intermitente cada tercer día y que no había consultado. En ese momento se le tomó gota gruesa, la cual se reportó como positiva para $P$. vivax. Luego de cinco días de hospitalización y tras presentar una evidente mejoría clínica, el paciente fue dado de alta.

\section{Discusión}

La malaria neonatal se define como aquella enfermedad que aparece en los primeros 28 días de vida posnatal (6). Esta definición no especifica si se toma a partir del inicio de los síntomas o al momento del diagnóstico. En nuestros pacientes hemos asumido el momento del inicio de los síntomas de la enfermedad actual como criterio de inclusión para considerarlos como casos neonatales.

El paludismo en este período de la vida incluye la posibilidad de un mecanismo de transmisión del parásito al neonato, tanto en forma vectorial como vertical (transplacentaria) (6). Esta diferenciación comienza con la verificación del cumplimiento de ciertos criterios, tomados de Henry (12), que puntualizan la transmisión vertical, así: antecedente materno de malaria gestacional por la misma especie de Plasmodium que se encontró en el neonato; obtención de aquél en sangre de cordón umbilical; antecedente materno de estancia en zona endémica o transfusión durante el embarazo; y demostrar en la zona endémica la presencia del parásito en los primeros siete días de vida postnatal para los casos de $P$. falciparum y hasta los 21 días para las demás especies.

De acuerdo con estos criterios, en el presente reporte solamente el paciente 4 se aproxima a esta definición de caso. En los demás, si bien presentaron un paludismo neonatal, no se pudo concluir con exactitud acerca de su mecanismo de transmisión.

Para hacer diagnóstico de malaria congénita es necesario realizar a los hijos de madres que sufrieron malaria en la gestación pruebas de laboratorio intraparto (gotas gruesas de sangre de cordón umbilical, placenta y sangre periférica del recién nacido) y seguimientos semanales clínicos y con gotas gruesas durante el primer mes de vida (10). Aunque tres de los pacientes tuvieron partos institucionales y en dos de ellos había antecedente materno de paludismo, en ningún caso se realizaron los procedimientos mencionados. Éstos no están incluidos ni en las guías de atención de la malaria ni en las de atención perinatal en nuestro país. Es posible que su escaso reporte se deba más a las deficiencias de su vigilancia epidemiológica, que a su baja frecuencia.

Llama la atención que los pacientes incluidos en este reporte presentaron cuadros clínicos graves, lo cual permitió identificarlos en el marco de la red de vigilancia y de asistencia. Sin embargo, no existe en el sistema sanitario actual una forma de identificar casos asintomáticos o leves y, por lo tanto, éstos ni se incluyen ni se describen.

Algunos aspectos maternos se han asociado con mayor posibilidad de paludismo en el neonato: baja número de partos, antecedente de fiebre e infecciones genitales en el último trimestre del embarazo y nivel socioeconómico bajo. Dos estudios realizados en Nigeria destacan la mayor frecuencia de la enfermedad en hijos de primigrávidas con antecedentes de fiebre $(5,6)$. En nuestro reporte todas las maternas refirieron fiebre durante el embarazo y en dos de ellas se hizo diagnóstico de malaria durante la gestación. El $50 \%$ estaban en su primera o segunda gestación y un porcentaje similar eran menores de 20 años, lo que corrobora el reporte de una asociación alta de infecciones neonatales en madres adolescentes (13).

Todos los pacientes de este reporte se caracterizaron por presentar síntomas y signos compatibles con una enfermedad infecciosa grave: fiebre, palidez intensa y hepatoesplenomegalia. Si bien es cierto que la fiebre es una de las manifestaciones más frecuentes del paciente pediátrico y que hace parte de los mecanismos de defensa frente a los agentes infecciosos, independientemente del estado clínico de la enfermedad, en el período neonatal siempre se debe considerar como manifestación de 
enfermedad grave (14), dadas las condiciones de inmadurez inmunológica en esta etapa de la vida y su frecuente asociación con cuadros clínicos ominosos, como la sepsis neonatal $(9,15)$. Estas manifestaciones se han descrito también en los casos de paludismo congénito ocurridos en zonas de baja endemia y transmisión inestable, en donde la materna no adquiere una inmunidad protectora adecuada contra Plasmodium, dejando al neonato más susceptible de enfermar gravemente (10).

Las cifras de hemoglobina y hematocrito estuvieron muy por debajo de los valores normales para la edad (hasta 9,0 mg/dl si consideramos la anemia fisiológica del lactante), y en dos pacientes se asociaron a manifestaciones clínicas que reflejaban la repercusión metabólica de la anemia (taquicardia, taquipnea, soplo cardiaco y cianosis). En los pacientes a quienes se solicitó perfil de bilirrubinas (tres de cinco), se encontró hiperbilirrubinemia mixta (bilirrubina total $>2,0 \mathrm{mg} /$ dl y bilirrubina directa $>0,5 \mathrm{mg} / \mathrm{dl}$ ), explicable tanto por el proceso hemolítico característico de la malaria como por la respuesta inflamatoria sistémica, inductora de disfunción hepática. Todos los pacientes presentaron algún grado de trombocitopenia, pero sólo en uno de esta serie correspondió a una trombocitopenia grave que incluso se acompañó de sangrado (16).

Teniendo en cuenta los criterios de enfermedad muy grave en el menor de dos meses propuestos por la Organización Mundial de la Salud (OMS), en su estrategia Atención Integrada a la Enfermedades Prevalentes de la Infancia (AIEPI), los cinco pacientes cumplían, al menos, con uno de los criterios y en cuatro de ellos se encontraron dos o más de los mismos (17). Si consideramos las normas del consenso internacional para la clasificación de la sepsis y el choque séptico en pediatría, el paciente 2 podría clasificarse como en sepsis y el paciente 5 como en sepsis grave (18).

Por otro lado, si se utilizan los criterios de paludismo grave promovidos por la OMS (19), dos pacientes cumplirían el criterio de anemia grave (hematocrito menor de 15\% o hemoglobina menor de $5 \mathrm{~g} / \mathrm{dl}$ ), dos, el de complicaciones hepáticas e ictericia (ictericia o bilirrubina total $>3 \mathrm{mg} / \mathrm{dl}$ ), uno, el de hemorragias espontáneas o coagulación intravascular diseminada y otro, el de insuficiencia respiratoria; sin embargo, hay que aclarar que estos criterios son rígidos, corresponden a pacientes muy gravemente enfermos y no han sido diseñados para la población neonatal y su uso en los casos de neonatos debe replantearse.

El tratamiento de la malaria depende de la especie parasitaria y del estado clínico del paciente. Cuando los neonatos cursen con una malaria no complicada, el esquema de tratamiento depende de la especie parasitaria identificada, con los mismos esquizonticidas sanguíneos y dosis que las utilizadas en el adulto o niños de mayor edad, pero con la precaución de evitar el uso de primaquina, debido a sus efectos adversos identificados en esta población específica, como la anemia hemolítica y la hepatotoxicidad. El medicamento base para aquellos pacientes con criterios de gravedad, independientemente del agente etiológico, es la quinina, supervisando la administración del medicamento idealmente en una unidad de cuidados intensivos $(19,20)$. Aunque los cinco pacientes correspondían a casos de paludismo neonatal grave, ninguno de ellos recibió el esquema antipalúdico recomendado para ésta.

Con base en todo lo anterior, podemos concluir que el presente reporte se trata de casos de malaria neonatal grave por Plasmodium vivax, especie que habitualmente no se considera como productora de complicaciones. Sin embargo, si tenemos en cuenta que en la etapa neonatal toda infección tiene mayor riesgo de complicarse y ocasionar la muerte, recomendamos ser más acuciosos en el diagnóstico y tratamiento adecuados y oportunos de estos pacientes, lo cual debe partir de mejorar la sospecha diagnóstica de esta entidad, de una vigilancia constante del paludismo durante la gestación y de un seguimiento sistemático a los neonatos en riesgo.

\section{Agradecimientos}

Los autores del presente trabajo quieren agradecer al personal de Archivo Clínico de los Hospitales de Turbo y Apartadó que facilitaron el acceso a las historias clínicas de los casos. 


\section{Conflicto de intereses}

No existe conflicto de intereses entre los autores, las instituciones financiadoras y el contenido del presente trabajo.

\section{Financiación}

El presente trabajo fue financiado por el Grupo Malaria de la Universidad Antioquia y por el Instituto Colombiano de Medicina Tropical del Instituto de Ciencias de la Salud (ICMT-CES).

\section{Referencias}

1. Breman JG, Alilio MS, Mills A. Conquering the intolerable burden of malaria: what's new, what's needed: a summary. Am J Trop Med Hyg. 2004;71(Suppl 2):1-15.

2. Dirección Seccional de Salud de Antioquia. Eventos de salud pública. [Consultado: mayo de 2007]. Disponible en: http://www.dssa.gov.co/htm/event_1.html

3. Desai M, ter Kuile FO, Nosten F, McGready R, Asamoa K, Brabin B, et al. Epidemiology and burden of malaria in pregnancy. Lancet Infect Dis. 2007;7:93-104.

4. Obiajunwa PO, Owa JA, Adeodu OO. Prevalence of congenital malaria in Ile-Ife, Nigeria. J Trop Pediatr. 2005;51:219-22.

5. Orogade AA. Neonatal malaria in a mesoendemic Malaria area of northern Nigeria. Ann Afr Med. 2004;3:170-3.

6. Runsewe-Abiodun IT, Ogunfowora OB, Fetuga BM. Neonatal malaria in Nigeria, a 2 year review. BMC Pediatr. 2006;6:19-24.

7. Alves MJ, Rangel O, Souza SS. Malaria in region of Campinas, Sao Paulo, Brasil, 1980 a 1994. Rev Soc Bras Med Trop. 2000;33:53-60.

8. Fernández RD, García Y, Alger J. Malaria y embarazo: observaciones clínico-epidemiológicas en dos zonas geográficas de Honduras. Rev Med Hondur. 2001;69:8-18.

9. Marques HH, Vallada MG, Sakane PT, Boulos M. Congenital malaria. case reports and a brief review of literature. J Pediatr (Rio J). 1996;72:103-5.
10. Piñeros JG. Malaria congénita. En: Carmona-Fonseca J, editor. Tópicos selectos de infectología. Medellín: Universidad de Antioquia; 2002.

11 Reproductive, Maternal and Child Health Erupean Regional Office World Health Organization. Definitions and indicators in family planning, maternal and child health and reproductive health used in the regional office for Europe. Geneve: WHO; 2001.

12. Henrys D. A propósito de un caso de paludismo congénito en Thomonde, Haití. Acta Médica Dominicana. 1983;6:216-8.

13. Lawn JE, Cousens S, Zupan J. 4 million neonatal deaths: When? Where? Why? Lancet. 2005;365:891900 .

14. Jaramillo-Bustamante JC. Malaria congénita. En: Departamento de Microbiología y Parasitología, editores. Tópicos selectos de infectología. Medellín: Universidad de Antioquia; 2006.

15. Hagmann S, Khanna K, Niazi M, Purswani M, Robins EB. Congenital malaria, an important differential diagnosis to consider when evaluating febrile infants of immigrant mothers. Pediatr Emerg Care. 2007;23:326-9.

16. Behrman RE, Kliegman R, Jenson HB. Nelson textbook of pediatrics. 16th Edition. Philadelphia: W.B. Saunders; 2000.

17 Organización Panamamericana de la Salud. Atención Integrada a las Enfermedades Prevalentes de la Infancia (AIEPI). Curso clínico para profesionales de la salud. Bogotá, D.C.: Ministerio de la Protección Social; 2005.

18. Goldstein B, Giroir B, Randolph A. International pediatric sepsis consensus conference: Definitions for sepsis and organ dysfunction in pediatrics. Pediatr Crit Care Med. 2005;6:2-8.

19. World Health Organization. Severe falciparum malaria. Trans Roy Soc Trop Med Hyg. 2000;94 (Suppl.1):s1/2

20. Ministerio de Salud. Guía de atención de la malaria. Diario Oficial. Edición 43956. Santa Fe de Bogotá: Ministerio de Salud; 2000. p.173-84. 